\title{
The reaction of a patient with suspicious retroverted epiglottis to an anesthetic
}

\author{
Hyo Myoung Lee, Se Hun Kim, and Seong Su Kim \\ Department of Anesthesiology and Pain Medicine, University of Ulsan College of Medicine, Gangneung Asan Hospital, Gangneung, Korea
}

Epiglottic abnormalities are uncommon, but, when they exist, they can hinder successful endotracheal intubation (ETI). Several reports have been published that address the issue of airway management for patients with epiglottic abnormalities, but no report concerning a case of retroverted epiglottis could be found in the literature. Therefore, we report a case in which we suspect that intubation failed due to a retroverted epiglottis.

A 49-year-old woman ( $159.3 \mathrm{~cm}$ tall and $39.6 \mathrm{~kg}$ ) was diagnosed with in situ carcinoma of the cervix. She was scheduled for laparoscopic abdominal hysterectomy with bilateral salpingo-oophorectomy. She had histories of pharyngolaryngitis and acute epiglottitis 25 years ago and had recently suffered from globus pharyngeus (GP), odynophagia, sore throat, hoarseness, and generalized weakness.

We recommended that she see an otolarygologist, and it was found that she had a narrow airway, a posteriorly-curling epiglottis, and vocal cords that could not be observed with laryngoscopy (Fig. 1). The otolarygologist's diagnosis was that the patient had a retroverted epiglottis and recommended conventional ETI with a small-sized endotracheal tube (ETT). Other pre-operative airway physical examinations, laboratory findings, her ECG, and her chest X-ray were all normal.

Anesthesia was induced with the intravenous administration of $200 \mathrm{mg}$ of thiopental sodium, $40 \mathrm{mg}$ of lidocaine, and 30 $\mathrm{mg}$ of rocuronium. After mask ventilation with $\mathrm{O}_{2}(3 \mathrm{~L} / \mathrm{min})$, $\mathrm{N}_{2} \mathrm{O}$ (3 L/min), and sevoflurane (3.0 vol\%), we attempted ETI with a 6.5-mm internal diameter ETT under direct laryngoscope. We judged the airway as class II by Cormack and Lehane's laryngoscopic airway classification. We watched far and small vocal cord, but ETT wasn't introduced into the trachea with resistance. We ventilated with a mask with $100 \%$ oxygen and attempted intubation again, first with a 6.0 $\mathrm{mm}$ internal diameter ETT and then with a 5.5-mm ETT, but we could not introduce either ETT. After the three trials, the tissue surrounding the airway was swollen, and we could no longer see the vocal cords. Fortunately, the mask ventilation was successful, and the pulse oximeter showed $100 \%$ oxygen

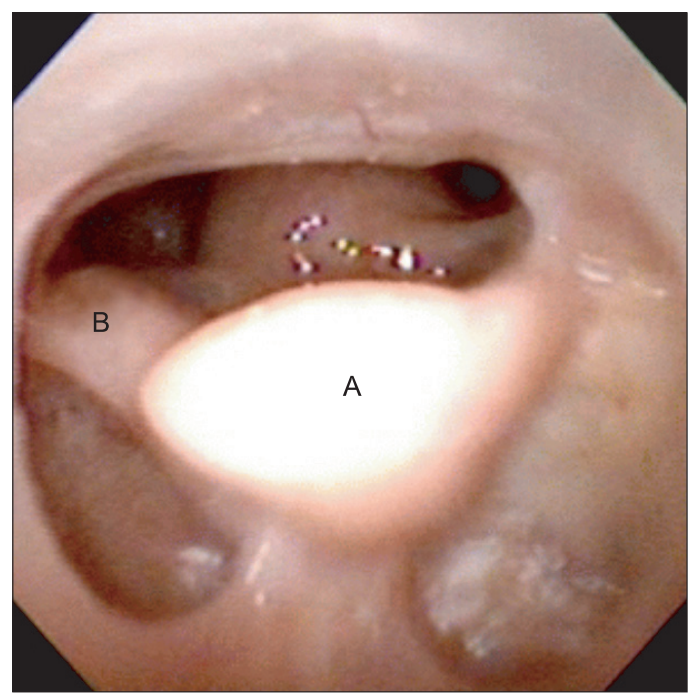

Fig. 1. Laryngoscopic examination. A: epiglottis, B: arytenoid cartilage.

Corresponding author: Hyo Myoung Lee, M.D., Department of Anesthesiology and Pain Medicine, University of Ulsan College of Medicine, Gangneung Asan Hospital, Bangdong-ri, Sacheon-myeon, Gangneung 210-711, Korea. Tel: 82-33-610-3114, Fax: 82-33-610-3409, E-mail: 05081110@naver.com

(c) This is an open-access article distributed under the terms of the Creative Commons Attribution Non-Commercial License (http:// creativecommons.org/licenses/by-nc/3.0/), which permits unrestricted non-commercial use, distribution, and reproduction in any medium, provided the original work is properly cited. 
saturation. Thus, we introduced \#3 LMA, but it did not fit her airway, and air leakage was observed. Ten milligrams of dexamethasone were administered intravenously to reduce edema. We decided to postpone the operation, and we administered $0.3 \mathrm{mg}$ of glycopyrrolate and $10 \mathrm{mg}$ of pyridostigmine to assist her in recovering from anesthesia. After she regained consciousness, she did not complain about any other symptoms in the recovery room, and she was transferred to the general ward. The next day, we decided to attempt unanesthetized intubation with a flexible fiberoptic bronchoscope (FOB). Both superior laryngeal nerves were blocked with $6 \mathrm{ml}$ of $2 \%$ lidocaine, and the oropharyngeal airway was anesthetized topically with $10 \%$ xylocaine. The FOB was introduced successfully into the trachea through the oral cavity. However, the smallest-sized tube (internal diameter of $5.5 \mathrm{~mm}$ ) could not pass the vocal cord. We terminated any further intubation trials and decided to operate using the trans-abdominal approach with combined spinal and epidural anesthesia, and an epidural catheter was introduced in the interspinous space at the L3-4 position. Ten milligrams of $0.5 \%$ heavy bupivacaine were injected through a $25 \mathrm{G}$ spinal needle at L4-5 intrathecal space, and $5 \mathrm{ml}$ of $2 \%$ lidocaine with $100 \mu \mathrm{g}$ of fentanyl 100 were injected through the epidural catheter. We confirmed that the sensory block had reached the T4 level, and a total abdominal hysterectomy with bilateral salpingooophorectomy was performed successfully under regional anesthesia. Her airway was evaluated by otolaryngologists with an FOB on the fourth day after the operation. There were no other changes in the airway compared with previous findings. The patient was discharged on the sixth day after the operation.

Currently, 'retroverted epiglottis' is not formal medical terminology. Most literature references refer to an 'anteriorlycurled epiglottis' as a 'retroverted epiglottis,' and others refer to a 'posteriorly-curled epiglottis' as a 'retroverted epiglottis.' There are very few reports that describes a posteriorly-curled epiglottis or an endotracheally-intruded epiglottis. This likely is due to the fact that epiglottic abnormalities rarely occur.

Globus hystericus (GP) is a relatively frequent clinical symptom (3-4\%) of unknown cause, and it is the most common symptom of a retroverted epiglottis. Patients who have epiglottic abnormalities suffer from GP, sore throat, and dysphagia [1]. In the case we have just described, the woman complained of GP and respiratory difficulty, and she had low body weight due to dysphagia. Therefore, when patients present with severe globus symptoms and low body weight, severe epiglottic abnormality should be considered.

The rate of incidence of retroverted epiglottis is unknown, as is the relationships between its anatomical variations and GP [1]. Inverted epiglottis has been described in some literature references. Agada et al. [1] reported four patients who presented with high GP and had abnormally-curled epiglottis tips that touched and indented the base of the tongue. Nakayama et al. [2] reported three patients who had complete epiglottic prolapse that obstructed the neoglottis after pexis as a postoperative complication of supracricoid laryngectomy with cricohyoidoepiglottopexy. They also reported that the three clinical factors that can be considered as causes of an inverted epiglottis are 1) extensive excision of the epiglottic petiole, 3) a low vallecula profile compared with the hyoid bone position, and 3) incorrect suturing to the remaining epiglottis during pexis. In addition to surgery and congenital abnormalities, other conditions that can cause an inverted epiglottis include inflammation and radiation therapy. In the case we described, the woman had frequent occurrences of sore throat after having acute epiglottitis 25 years ago, but no one has reported that acute epiglottitis can be a causative factor for the retroverted epiglottis condition. Therefore, it is difficult to discern exactly whether she had a congenital abnormality or an acquired abnormality.

In general, difficult intubation means that intubation takes more than three trials or more than 10 minutes [3]. The overriding rule, however, is that there be sufficient oxygenation between the trials, at least one or more of the change on the next trial [4]. In the case we described, we were unsuccessful in our intubation attempts after trying three times, and we were unable to see the patient's vocal cords due to traumatic swelling. We chose not to make additional attempts to avoid the possibility of creating the critical situation involving the 'cannot-intubate, cannotventilate state.' Thus, we considered the use of unanesthetized intubation with an FOB than conventional intubation because we were able to avoid hypoxia by allowing the patient to maintain self respiration, to see the patient's vocal cords, and to provide a safe way for the patient to recover.

In conclusion, anesthesiologists should induce anesthesia only after systematic, pre-operative preparation in cases that involve epiglottic abnormalities. They also should consider regional anesthesia within practical limits and try unanesthetized intubation using an FOB or ventilation through LMA if general anesthesia is essential. Also, emergent tracheostomy or cricothyrotomy must be prepared to avoid fatal ventilation failure.

\section{References}

1. Agada FO, Coatesworth AP, Grace AR. Retroverted epiglottis presenting as a variant of globus pharyngeus. J Laryngol Otol 2007; 121: 390-2.

2. Nakayama M, Okamoto M, Seino Y, Miyamoto S, Hayashi S, Masaki, T, et al. Inverted epiglottis: a postoperative complication of supracricoid laryngectomy with cricohyoidoepiglottopexy. Auris 
Nasus Larynx 2010; 37: 609-14.

3. Yang CM, Lim YS, Cho CK, Kwon HU, Kang PS, Yang CW. Experience of laryngeal tube insertion in a patient of expected difficult intubation. Korean J Anesthesiol 2007; 52: 457-60.
4. Park CH, Kim HY, Rho WS, Kim BI, Kim JK, Chung JY. Difficult intubation due to low located and right deviated thyroid cartilage. Korean J Anesthesiol 2006; 51: 742-5. 